\title{
METASTABLE ONE- AND TWO-ELECTRON DONOR STATES IN GaAs AND $\mathrm{CdF}_{2}$
}

\author{
S. Bednarek and J. Adamowski \\ Faculty of Physics and Nuclear Techniques \\ Technical University of Mining and Metallurgy (AGH) \\ Al. Mickiewicza 30, 30-059 Kraków, Poland
}

\begin{abstract}
The strongly localized one-electron $\left(D^{0}\right)$ and two-electron $\left(D^{-}\right)$donor states are considered with the lattice deformation around the donor center taken into account. For $\mathrm{GaAs}$, the donor energy levels have been calculated as functions of the hydrostatic pressure. The calculated energy positions and pressure coefficients agree with the experimental data. It is shown that the interaction with phonons reduces the probability of radiative transitions between the states of different localization and leads to the metastability of shallow-level donor states with respect to the $D^{-}$state in GaAs and both the states $\left(D^{0}\right.$ and $\left.D^{-}\right)$in $\mathrm{CdF}_{2}$.
\end{abstract}

PACS numbers: 71.55.-i

In a semiconductor compound, three types of donor states can be formed on the same impurity atom [1]. These are: the weakly localized (hydrogen-like) states $\left(d^{0}\right)$ of shallow energy levels, strongly localized one-electron states $\left(D^{0}\right)$, and strongly localized two-electron states $\left(D^{-}\right)$. These three types of donor states were observed in GaAs under hydrostatic pressure [1,2] and in $\mathrm{Al}_{x} \mathrm{Ga}_{1-x} \mathrm{As}$ [3]. The one-electron donor states of different localization, i.e., $d^{0}$ and $D^{0}$, were observed in $\mathrm{CdF}_{2}$ [4]. The authors [5] elaborated a unified theoretical method which allows us to describe the one-electron donor states of different localization. It has been shown [5] that the incorporation of the electron-phonon interaction is of special importance in understanding the properties of these states. In the present paper, the approach [5] is extended to the strongly localized two-electron $\left(D^{-}\right)$ donor states. We have calculated the energy levels and probabilities of radiative transitions between the donor states of different localization. In particular, we discuss the mechanism of metastability of the donor states.

The calculations have been performed in the frame of one-band approximation with the model conduction-band structure [5]. We have taken into account the interaction with LO phonons via the Frohlich coupling and with LA phonons via the deformation potential. We consider the donor center creating the long-range (Coulomb) and short-range fields with one and two electrons bound to this center. The wave functions and energies of the considered donor states have been obtained 
by variational means. For GaAs, we have calculated the electronic energy levels of the donor states $d^{0}, D^{0}$, and $D^{-}$as functions of the hydrostatic pressure. The influence of pressure has been included in the same manner as in our previous papers [5]. The results are shown in Fig. 1a.
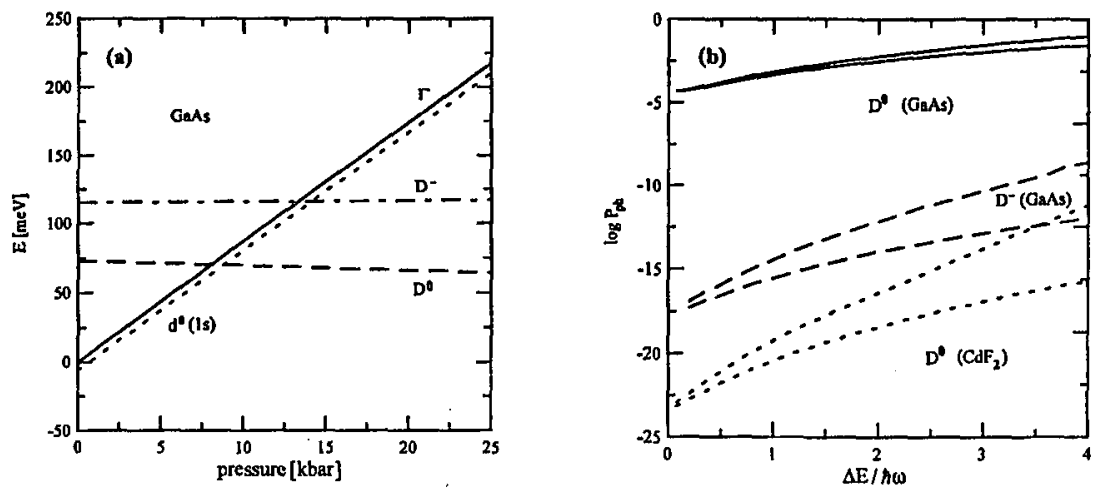

Fig. 1. (a) Calculated energy levels of the strongly localized donor states $D^{\circ}$ and $D^{-}$ in $\mathrm{GaAs}$ as functions of the hydrostatic pressure. The $\Gamma$ conduction-band minimum and the shallow energy level $d^{0}$ are also shown for comparison. The curves correspond to the energy calculated per one electron. (b) Lower and upper bounds on the phonon factor of the probability of leaving the weakly localized donor state $d^{0}$ with radiative transitions to the strongly localized states $D^{0}$ and $D^{-}$as functions of the energy separation $\Delta E$ between the initial state and final ground state.

In the present work, the variational calculations have been performed with the help of the one-element trial wave function. This means that the results of Fig. 1a provide the correct upper bounds for the energy of the ground state only. At low pressure $(p \leq 8.5 \mathrm{kbar})$, the $d^{0}$ is the ground state of the donor. The $D^{-}$ is the ground state of the two-electron system if the two electrons bound to the donor center possess a lower energy than the system composed of the one electron in the $D^{0}$ state and the second - in the ionized state (conduction band), i.e.

$$
2 E_{D-} \leq E_{D^{0}}+E_{\Gamma} \text {. }
$$

This inequality is fulfilled for the pressure $p \geq 20 \mathrm{kbar}$. Just in this pressure regime, the energy level associated with the metastable DX state is located in the gap [6]. For other values of pressure, the corresponding curves can be treated as extrapolations. At the ambient pressure, the strongly localized $D^{0}$ and $D^{-}$states, are resonant with the conduction band. The energy levels of these states calculated for zero pressure are located at $75 \mathrm{meV}$ and $115 \mathrm{meV}$, respectively, in agreement with experimental data [2]. The calculated pressure coefficients $(-9 \mathrm{meV} / \mathrm{kbar})$ for both the states are nearly equal to each other and also agree with the experimental values [2].

Having at our disposal the wave functions obtained from the variational calculations, we can estimate the probabilities of radiative transitions between the donor states. Let us consider the case in which the strongly localized state, i.e. 
$D^{0}$ or $D^{-}$, is the ground state of the donor. We assume the initial state to be the weakly localized donor state $\left(d^{0}\right)$ of shallow energy level and calculate the probability $P$ of leaving this state by the electron. For this purpose, we consider the spontaneous radiative transitions to all the final states of lower energy, which are the many-phonon states corresponding to the electron in the strongly localized donor state $\left(D^{0}\right.$ or $\left.D^{-}\right)$. The energy levels of these states form the quasi-continuous energy band arising from LA phonons. The probability $P$ can be approximated by the product of the electronic part $P_{\mathrm{el}}$ and the phonon part $P_{\mathrm{ph}}$, i.e.

$$
P=P_{\mathrm{el}} P_{\mathrm{ph}} \text {. }
$$

Due to the appearance of low-energetic LA phonons, it is not possible to calculate the phonon factor $P_{\mathrm{ph}}$ exactly. Nevertheless, we have succeeded in receiving the useful estimates of $P_{\mathrm{ph}}$ from below and from above. Figure 1b shows the calculated lower and upper bounds on the phonon part of the transition probability as functions of the energy separation between the initial and ground states. The solid curves correspond to both the bounds for the ground state being the $D^{0}$ donor state in GaAs, the dashed curves - to those for the $D^{-}$state in GaAs, and the dotted curves - to those for the $D^{0}$ state in $\mathrm{CdF}_{2}$. We can observe that the effect of the phonon factor on the total transition probability is the largest for small energy separations. If the $D^{0}$ is the ground state, $P_{\mathrm{ph}}$ reduces the transition probability by the factor $10^{-4}$ in GaAs and by $10^{-24}$ in $\mathrm{CdF}_{2}$.

The reduction of transition probabilities results from the great difference of the lattice deformation around the donor center between the states of different electron localization, which causes the considerable decrease in the overlaps between the phonon parts of the wave functions for the initial and final states. In the case of two-electron strongly localized $D^{-}$states, the difference of the lattice distortion with respect to the weakly localized states becomes much larger. Therefore, the probability of radiative transitions from the weakly localized states undergoes further reduction. The results of the present paper show that the probability of direct transitions of the electrons from the weakly localized donor states or from the conduction band to the $D^{-}$state is limited by the phonon factor smaller than $10^{-10}$ (even in GaAs). Therefore, we can expect the occurrence of the metastability of the weakly localized donor states and delocalized conduction band states with respect to the $D^{-}$states in $\mathrm{GaAs}$. In $\mathrm{CdF}_{2}$, the corresponding metastability will appear with respect to both the strongly localized states $D^{0}$ and $D^{-}$.

If the separation between the energy levels increases, the transition probability $P_{\mathrm{ph}}$ increases as well due to the increase in the number of many-phonon final states. For the energy separation of several LO-phonon energies, $P_{\text {ph }}$ becomes larger by several orders of magnitude. This means that the metastability (provided it appeared at small energy separation) disappears. In order to show this effect we exploit the lower bound on the transition probability. The upper bound on this probability has allowed us to draw conclusions on the appearance of the metastability.

The present calculations are relevant to the donor center at a substitutional site. For GaAs, the results received for the energy positions and pressure coefficients of the $D_{0}$ and $D^{-}$donor states agree with the experimental results [2] for the DX center. This provides a strong suggestion that in GaAs under hydrostatic 
pressure the DX center can be identified with the substitutional $D^{-}$state. The results of the $a b$ initio calculations [7] lead to the interpretation of the DX centers as the $D^{-}$states with the donor center at the interstitial site. Such a configuration can be the most favorable one among the two-electron states in $\mathrm{Al}_{x} \mathrm{Ga}_{1-x} \mathrm{As}$ (for $x>0.22$ ). Then, the impurity atom is shifted into the interstitial position with broken bonds.

The present approach is based on the phonon picture of the lattice deformation, which means that we assume small displacements of the ions. Taking into account the shift of the impurity center into the interstitial position goes beyond the applicability of the present model. Therefore, we cannot estimate the energy of this configuration in our model. However, due to the large lattice deformation resulting from the bond breaking, we can expect the coexistence of the two types of $D^{-}$donor states: one state with the donor center at the substititional position and the second - at the interstitial position. This conclusion requires a future experimental verification.

\section{References}

[1] J.E. Dmochowski, R.A. Stradling, Jpn. J. Appl. Phys. 32, Suppl. 32-1, 227 (1993).

[2] M. Baj, L.H. Dmowski, T. Słupiński, Phys. Rev. Lett. 71, 3529 (1993); P.J. van der Wel, P. Wiśniewski, T. Suski, J. Singleton, C. Skierbiszewski, L.J. Giling, R. Warburton, P.J. Walker, N.J. Mason, R.J. Nicholas, M. Eremets, J. Phys., Condens. Matter 5, 5001 (1993).

[3] J.E. Dmochowski, R.A. Stradling, J. Sly, D.J. Dunstan, A.D. Prins, A.R. Adams, Acta Phys. Pol. A 87, 457 (1995).

[4] U. Piekara, J.M. Langer, B. Krukowska-Fulde, Solid State Commun. 23, 583 (1977).

[5] S. Bednarek, J. Adamowski, in: The Physics of Semiconductors, Ed. D.J. Lockwood, World Scientific, Singapore 1995, p. 2335; S. Bednarek, J. Adamowski, Phys. Rev. $B$ 51, 4687 (1995).

[6] M. Mizuta, M. Tachikawa, H. Kukimoto, S. Minomura, Jpn. J. Appl. Phys. 24, L143 (1985).

[7] D.J. Chadi, K.J. Chang, Phys. Rev. B 39, 10063 (1989); J. Dąbrowski, M. Scheffler, Mater. Sci. Forum 83-87, 735 (1992). 